\title{
3 Research Square \\ The diagnostic value of 3D-ASL in vertebral artery stenosis among individuals at risk of stroke
}

\section{DanDan Xu}

Quanzhou Hospital Afiliated of Fujain Medical University

\section{XinMing Huang}

Fujian Medical University Union Hospital

\section{GuoEn Cai}

Fujain Medical University Union Hospital

\section{XiaoXia Yang}

Fujian Medical University Affiliated First Quanzhou Hospital

\section{YingQing Wang}

Fujain Medical University Union Hospital

\section{Bin Sun}

Fujian Medical University Union Hospital

\section{XiaoChun Chen}

Fuajin Medical University Union Hospital

QinYong Ye ( $\square$ unionqyye@163.com )

Fujian Normal University

\section{Research article}

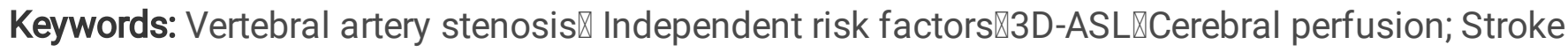

Posted Date: August 28th, 2019

DOI: https://doi.org/10.21203/rs.2.13638/v1

License: (c) (i) This work is licensed under a Creative Commons Attribution 4.0 International License.

Read Full License 


\section{Abstract}

Background: This study explored the predictive value of 3D-ASL for vertebral artery stenosis (VAS) in individuals at risk of stroke. Methods: A total of 43 participants at risk of stroke were enrolled in our study. All participants underwent 3D-ASL and carotid artery computed tomography angiography (CTA) or cervical vascular ultrasound. Cerebral blood flow (CBF) was measured in the left and right frontal lobes, temporal lobes, parietal lobes, occipital lobes, basal ganglia, thalamus, cerebellum, and hippocampus. Calculate the CBF BI (CBF BI means: The absolute value of the difference in cerebral blood flow in the corresponding brain regions on the left and right sides of the enrolled personnel) of the corresponding brain regions of the left and right between the VAS group and the non-VAS group respectively. To evaluate the predictive value of 3D-ASL for vascular stenosis in participants at risk of stroke, we used two-sample t-tests to determine if there were significant differences in BI. Results: Among 43 participants at risk of stroke we recruited, 20 participants with vertebral artery stenosis (the VAS group) and 23 without vertebral artery stenosis (the non-VAS group). There were significant differences in BI of the occipital lobe between the VAS group and the non-VAS group $(p<0.05)$. According to the occipital lobe BI value, the receiver operating characteristic curve (ROC) demonstrated best diagnostic limits of $10.46 \mathrm{ml} /(100 \mathrm{~g} \times \mathrm{min})$. Conclusion: Using 3D-ASL to detect cerebral blood flow and calculate the difference of bilateral occipital cerebral blood flow which can help to diagnose vertebral artery stenosis in high risk population of stroke.

\section{Background}

Stroke is the second leading cause of death worldwide, behind only ischemic heart disease. Aside from the high mortality rate, the morbidity associated with stroke has huge patient-level and societal impacts[1, 2]. Posterior circulation ischemic stroke involves the vertebrobasilar arterial system ischemia, cerebrovascular occlusion, brain tissue ischemia, and hypoxia necrosis; it accounts for $20 \%$ of strokes [3]. The main symptoms in affected patients are dizziness, vomiting, diplopia, ataxia, coma, difficulty breathing, and even death [4]. The most common cause of the disease is vertebrobasilar arterial stenosis caused by atherosclerosis, but for many patients, difficulties in locating the causative vascular lesions at the time of presentation to hospital lead to delays in clinical interventions. Therefore, early diagnosis of vertebral artery stenosis and early intervention are key determinants of clinical rescue and quality of life after posterior circulation ischemic stroke.

At present, the imaging examinations that can assist in the diagnosis of vertebral artery stenosis mainly include cervical vascular ultrasound, magnetic resonance angiography (MRA), computed tomography angiography (CTA), digital subtraction technique (Digital subtraction angiography, DSA), etc. It helps to determine the type of aortic arch and assist in judging the nature of the lesion of arterial stenosis. However, because it is an invasive examination, there may be contrast nephropathy and puncture complications. Therefore, it is often necessary to make a preliminary diagnosis after non-invasive examination to determine whether to perform DSA examination, which affects its wide application in the clinic. Arterial spin labeling (ASL), which emerged as a magnetic resonance imaging (MRI) technique in 1992 [5], is a non-invasive, safe, reliable, technically straightforward, and low-cost method for assessing 
$\mathrm{CBF}[6]$. ASL technology has the advantages of high reproducibility, non-invasiveness, didn't need to inject contrast agents[7]. Furthermore, 3D-ASL technology can further improve signal-to-noise ratio, reduce motion artifacts, and improve image quality[8]. It is a continuous marker imaging based on a new RF platform, which overcomes the shortcomings of low signal-to-noise ratio and uneven perfusion, and improves the contrast and reliability of perfusion imaging. Owing to these advantages, the technique has steadily gained popularity and is now in widespread use in clinical practice. The basic principle is that the arterial blood is magnetized to mark protons before it flows into the tissue. Therefore, it can be regarded as a tracer technique for estimating tissue perfusion using water as a natural endogenous tracer, which generates marks by magnetizing many protons in the blood with radio-frequency pulses. After the marking period and the inflow period, images are obtained using fast acquisition techniques, such as echo planar imaging, gradient and spin echo imaging (GRASE), or 3D fast spin echo imaging using a spiral stacking method. Two images are required-one is the image of the arterial blood that is magnetized, that is, the image of the marker; the other is the image of the arterial blood that is not magnetized, that is, the control image. The difference in signal between the marker image and the control image is proportional to the amount of magnetization that is converted and delivered to the tissue[9]. If all labeled blood reaches the image voxel during the image acquisition period, the signal difference will be proportional to the brain blood volume (CBF) [10]. The reduction or abnormality of cerebral blood perfusion is the pathological basis and main diagnostic basis of ischemic cerebrovascular disease. ASL technology can be used for detecting the reduction and disappearance of early perfusion and calculating CBF values, which can be important reference values for the clinical diagnosis and treatment of hemodynamic changes in cerebrovascular diseases[11]. In view of this, in this paper we used 3D-ASL to study the predictive value of asymptomatic vertebral artery stenosis.

To our knowledge, there are few published reports on the use of three-dimensional (3D)-ASL to predict vertebral artery stenosis. We used 3D-ASL to quantify cerebral perfusion among study participants with vertebral artery stenosis and analyzed differences in global cerebral perfusion and local perfusion distribution between such individuals and healthy controls. This analysis allowed us to evaluate the predictive value of 3D-ASL in vertebral artery stenosis, which may provide a reference for early identification and treatment of lesions causing posterior stroke.

\section{Methods}

\section{Study subjects}

We included 43 participants at risk of stroke in this study, which was conducted from December 2016 to December 2017 at the Department of Neurology, Union Hospital of Fujian Medical University. Potential participants were considered at risk of stroke if they fulfilled more than three of the following criteria: a history of hypertension ( $\geq 140 / 90 \mathrm{mmHg}$ ) or taking antihypertensive drugs, a diagnosis atrial fibrillation or valvular heart disease, smoking, abnormal dyslipidemia or unknown lipid profile, diagnosis of diabetes mellitus, sedentary lifestyle (not engaging in exercise $\geq 3$ times per week, $\geq 30$ minutes each time, and for more than 1 year; and not engaged in moderate to severe physical labor), body mass index (BMI) $\geq 26$ 
$\mathrm{kg} / \mathrm{m} 2$, family history of stroke, and age $>60$ years old [9]. Exclusion criteria were as follows: repeated history of head trauma, contraindications to MRI examination or contrast administration, inability or unwillingness to cooperate with imaging and study procedures, obvious imaging artifacts affecting interpretation, history of brain tumors, hydrocephalus, cerebral infarction, cerebral hemorrhage, or diagnosis that may affect other brain lesions. A total of 43 personnel was enrolled, of which 20 participants with vertebral artery stenosis (the VAS group) and 23 without vertebral artery stenosis (the non-VAS group). All subjects provided written informed consent before participating in the study. This study was approved by our hospital's institutional review board.

\section{Study procedures}

All subjects underwent 3D-ASL and either carotid CTA or cervical vascular ultrasound. They all had CBF measurements taken in the left and right frontal lobes, parietal lobes, temporal lobes, occipital lobes, basal ganglia, thalamus, hippocampus, and cerebellum (Figure 1).

\section{Magnetic resonance imaging}

We double-checked for contraindications to MRI examination before proceeding with each participant. Consumption of tobacco, alcohol, and illicit substances were not allowed before scanning (Tobacco, alcohol and illicit substances were banned 24 hours before the magnetic resonance examination.). All subjects were examined using a 3.0 Tesla magnetic resonance (MR) scanner (Discovery 750, General Electric Medical System, Milwaukee, WI, USA) and an 8-channel high-resolution head coil. Conventional MR images included axial T1 weighted images, axial T2 weighted images, axial T2-fluid-attenuated inversion recovery (FLAIR) images, axial T2* weighted images, axial diffusion weighted images, postcontrast axial positions, and coronal and sagittal T1-weighted image (T1WI). 3D-ASL imaging was performed by a spiral stack of pseudo-continuous ASL pulse sequences using 3D fast spin echo imaging sequences with background suppression. The planning of the label board is carried out at a fixed distance, and the planning process is as shown in supplementary figure1. The label duration is $1525 \mathrm{~ms}$. The parameters used were: 512 sampling points on 8 spirals, FOV $=24.0 \mathrm{~cm}$ (This study is a 3D scan, but not a layer-by-layer scan, so the FOV is not 3D), TR/TE $=4653 / 10.5 \mathrm{~ms}$, post-labeling delay (PLD) $=1525$ $\mathrm{ms}$ (The short PLD reflects the perfusion behavior, that is, the thickness of the perfused responsible blood vessels. The long PLD can reflect the true results of perfusion. This paper mainly studies the vertebral artery stenosis in high-risk stroke patients. The thickness is therefore more suitable for $1525 \mathrm{~ms}$ instead of a longer PLD), in-plane matrix $=128 \times 128$, bandwidth $= \pm 62.5 \mathrm{KHz}$, slice thickness $=4.0 \mathrm{~mm}$, inter-slice gap $=0 \mathrm{~mm}$, number of slices $=36$, average number $(\mathrm{NEX})=3$, acquisition time $=4 \mathrm{~min} 30 \mathrm{~s}$. The 3D-ASL data were transferred to an offline workstation (Advantage Workstation, AW4.5; GE Medical Systems). The CBF color map was then automatically processed using GE FuncTool software (The quantification model forms of the GE FuncTool software as supplementary figure2). Two neuroradiologists who were unaware of the final diagnosis analyzed all images in random order. Regions of interest (ROIs) were selected from the bilateral frontal, parietal, temporal, basal, thalamic, hippocampal, occipital, and cerebellar regions (In this study, we selected the ROI manually, and selected the fixed parts of the brains 
of the enrolled personnel for CBF calculation. The choice of ROI is carried out in WM). Using the selected ROIs, the mean CBF value in each brain region was measured (CBF value calculation is performed on the fixed parts of each brain lobe (left and right frontal lobe, parietal lobe, temporal lobe, occipital lobes, basal ganglia, thalamus, hippocampus and cerebellum) of the enrolled personnel. The selection of ROI is mostly in WM. Three locations were measured for each ROI, and the mean value was taken as representative; in order to minimize measurement error in the CBF analysis, a uniform ROI size [0.5 $\left.\mathrm{cm}^{2}\right]$ was maintained). After dividing the stroke-risk subjects into VAS and non-VAS groups, we made some calculations to evaluate the predictive value of 3D-ASL.The specific practices are as follows: Calculate respectively the VAS group, the non-VAS group difference of corresponding brain regions CBF BI (CBF BI means: The absolute value of the difference in cerebral blood flow in the corresponding brain regions on the left and right sides of the enrolled personnel. We have already added this part to the manuscript), and work out respectively the VAS group.

\section{Data analysis}

All data were processed and analyzed using the SPSS version 23.0 statistical software (IBM Corp., Armonk, NY, USA). Unless otherwise stated data are presented as mean \pm standard deviation (SD).To evaluate the predictive value of 3D-ASL for vascular stenosis in participants at risk of stroke, we used two-sample t-tests to determine if there were significant differences in mean CBF values among the groups and among the respective brain regions.

\section{Results}

\section{Comparison of general clinical data}

Among the 43 subjects in the stroke-risk group were 26 males and 17 females with a mean of $64.81 \pm$ 10.52 years. There were no significant differences in gender and age between the subjects at risk of stroke and the healthy controls $(P>0.05)$. Among the 20 cases in the VAS group, seven were of left-sided vertebral artery stenosis, 11 were right-sided, and two were bilateral; 10 of the VAS patients were male and 10 were female. The mean age of the VAS group was $62.70 \pm 11.77$ years. In the non-VAS group, there were 16 males and 7 females with a mean age of $67.04 \pm 9.01$. The stroke-risk group was divided into 20 cases of vertebral artery stenosis and 23 cases without vertebral artery stenosis (Table 1).

\section{Comparison of the value of BI between the VAS group and the non-VAS group}

The VAS group images were compared with those of the non-VAS group (Figure 2), and the perfusion of the posterior circulation brain region (especially the occipital lobe) was significantly reduced in the 3DASL images of the VAS group. The CBF values of the brain regions of the VAS group and the non-VAS group were measured and plotted as heat maps (Figure 3). The CBF values of the thalamus, basal ganglia, and hippocampus were higher than those of other brain regions. The BI values of the VAS group and the non-VAS group were measured and statistically analyzed (Table 2). There (Figure 4 and Table 2) show that statistically significant values were associated with the occipital lobe $(p<0.05)$. There were no 
statistically significant differences values related to the frontal lobes, parietal lobes, temporal lobes, cerebellum, hippocampus, basal ganglia, or thalamus.

Diagnostic value of the absolute value of the difference in occipital CBF in vertebral artery stenosis

The receiver operating characteristic curve (ROC) were obtained for evaluate the accuracy of the $\mathrm{BI}$ of the occipital lobe. The areas under the curve (AUCs) of occipital lobe BI was 0.887 and the areas under the curve (AUCs) of occipital lobe BI $\otimes 10$ was 0.860 with the cutoff values $10.46 \mathrm{ml} / 100 \mathrm{~g}$. The ROC curve is shown in Figure 5, and the cutoff values are shown in Table 3.

The effectiveness test of CBF difference in occipital lobe and cut-off value of various ratios

The cut-off values of the BI values of the occipital lobe were regrouped into two groups of patients. Parallel chi-square tests and binary logistic analysis verified the accuracy of the cut-off values and found that the cut-off values for $\mathrm{BI}(p=0.036)$ were statistically significant (Table 4$)$.

\section{Discussion}

Vertebral and basilar atherosclerotic diseases are common causes of stroke[12]. Insufficient blood supply to the vertebral and basilar arteries, due to the fact that associated symptoms overlap with the symptoms of many non-neurological diseases, lack specificity, so it is difficult to confirm the diagnosis by clinical manifestations alone. A study conducted at the New England Medical Center found that distal territory location, and basilar artery occlusive disease carried the poorest prognosis [10]. There is a high mortality rate associated with basilar artery occlusion and vertebral artery stenosis, and most survivors have severe permanent disability; therefore, early detection of vertebral artery stenosis is crucial[13].

Because of the low prevalence of severe carotid stenosis in the general population, screening for asymptomatic carotid stenosis is usually not necessary, except for among some subgroups in whom screening is worthwhile. According to results generated by the US Health Prevention Services Working Group, screening for asymptomatic carotid stenosis is not recommended for people at low risk of arterial stenosis because it may cause many false positives and lead patients to accept unnecessary angiography or surgery, which in turn can have some negative effects, such as contrast nephropathy and surgical complications[14]. In a study of 1348 subjects and a mean follow-up of 12.7 years, it was found that patients with a carotid intima-media thickness (IMT) of more than $1 \mathrm{~mm}$ had a $20 \%$ increase in stroke incidence compared with normal controls[15]. In another study of 5028 patients of different ethnicities, the carotid IMT thickened by $0.05 \mathrm{~mm}$ per year, associated with an significantly increasing incidence of stroke[16]. There is a correlation between cervical stenosis and stroke risk. Therefore, this study investigated vertebral artery stenosis in a high-risk population.

To the best of our knowledge, the study to investigate the use of 3D-ASL to predict vertebral artery stenosis is still few. According to our results, the cutoff of the BI value of the occipital lobe was 10.46, and the AUC was 0.887 . The cut-off value of occipital lobe BI value was regrouped into two groups of 
participants. Parallel chi-square testing and binary logistic analysis verified the accuracy of the cut-off values and found that the BI value of the occipital lobe $(p=0.036)$ were statistically significant.

Therefore, the vertebral artery stenosis can be predicted by calculating the BI value of the occipital lobe. In addition, vertebral artery stenosis causes a change in perfusion volume, but the change in perfusion volume has little effect on the labeling efficiency, or the amount of perfusion is not related to the labeling efficiency. If the degree of stenosis is very heavy or the vertebral artery itself is very small, the label will be inaccurate, and the subsequent measurement will not be accurate enough. The patients enrolled in this study have a small degree of arterial stenosis.

It is well known that about $80-90 \%$ of blood in the brain is supplied by the internal carotid artery system, and $10-20 \%$ is supplied by the vertebrobasilar arterial system. After the vertebral artery is issued by the subclavian artery, it passes through the transverse vertebral foramina at C6-C2, enters the brain from the occipital foramen, and merges at the junction of the pons and medulla, forming the basilar artery, which divides into bilateral posterior cerebral arteries (PCAs) at the top of the pons. Near the terminal bifurcation, the basilar artery emits the superior cerebellar artery (SCA), which supplies the cerebral pons and blood flow in the lateral region of the midbrain and the upper cerebellum. The superior cerebellar artery (SCA) and the inferior cerebellar anterior and posterior inferior cerebellar artery (PICA) from the basilar artery are mainly responsible for the blood supply to the cerebellum. The perforating artery of the PCA supplies the thalamus. The PCA also supplies the occipital cortex. Therefore, the results obtained in this study are consistent with the anatomical principles of occipital perfusion abnormalities caused by vertebral artery stenosis. The thalamus and cerebellum, which are perfused by the posterior circulation of the blood supply, did not demonstrate statistically significant predictive value. The significance of the occipital lobe may be related to the fact that some of the lobe is in the watershed area, and there are fewer anastomotic networks in the peripheral arteries. The demand for oxygen intake and energy metabolism is, therefore, higher and perfusion abnormalities are more likely to occur. Jiang Yun et al. found that single photon emission computed tomography (SPECT) brain blood perfusion imaging (to detect rCBF) can sensitively display occipital lobe lesions, and that this has a certain diagnostic value for ischemic lesions of the vertebrobasilar arterial system [17]. This also indirectly indicates that the occipital lobe is more sensitive to posterior circulation perfusion abnormalities than other sites, and is thus more useful for predicting vertebral artery stenosis.

The current research has several limitations. First, the sample size was limited to 43 subjects at risk of stroke. The number of entries in this paper is limit, it is inevitably a certain degree of deviation. Further research is needed to further expand the sample size increasing the reliability and repeatability of the conclusion. Second, because of the many factors that may influence the outcome of 3D-ASL, such as subject age, blood transit time, and PLD time selection, the fixed PLD used in this study may have introduced bias. A variable PLD should be used in future studies to validate our current perfusion methods. Third, because the incidence of vertebral artery stenosis is low in healthy people, and the number of people included in the study is small, if the healthy control group is included in the comparison, the results will be affected. Therefore, the study did not include healthy controls. Scope, but focus on the situation in the high-risk population of stroke. 


\section{Conclusions}

Using 3D-ASL to detect cerebral blood flow and calculate the difference of bilateral occipital cerebral blood flow can help to diagnose vertebral artery stenosis in high risk population of stroke.

\section{Abbreviations}

VAS: Vertebral artery stenosis; CTA: Computed tomography angiography; CBF: Cerebral blood flow; MRA: Magnetic resonance angiography; CTA: Computed tomography angiography; DSA: Digital subtraction angiography; ASL: Arterial spin labeling; GRASE: Gradient and spin echo imaging; BMI: Body mass index; FLAIR: T2-fluid-attenuated inversion recovery; T1WI: T1-weighted image; PLD: Post-labeling delay; AW: Advantage Workstation; ROI: Regions of interest; SD: Standard deviation; ROC: Characteristic curve; AUC: Areas under the curve; IMT: Intima-media thickness; PCA: Posterior cerebral arteries; SCA: Superior cerebellar artery; PICA: Posterior inferior cerebellar artery; SPECT: Single photon emission Computed tomography; Bl: Absolute value of the difference in cerebral blood flow in the corresponding brain regions on the left and right sides.

\section{Declarations}

\section{Acknowledgments}

Not applicable

\section{Competing interests}

The authors have disclosed that they have no significant relationships with, or financial interest in, any commercial companies pertaining to this article.

\section{Funding}

This work was supported by the Science Foundation of the Fujian Province, China (Grant No.2016Y01010083). The funders had proposed the study, and assisted in the analysis and interpretation of data, revising the manuscript for content, and also provided study supervision.

\section{Availability of data and materials}

The datasets generated and/or analysed during the current study are not publicly available due containing identifying medical information but deidentified data are available from the corresponding author on reasonable request. 


\section{Authors' contributions}

DDX and $\mathrm{XMH}$ managed the data, performed statistical analyses, drafted and revised the manuscript. QYY proposed the study, and assisted in the analysis and interpretation of data, revising the manuscript for content, and also provided study supervision. XCC, GEC, and XXY assisted with revising the manuscript and the acquisition of the data. YQW and BS assisted with analysis and interpretation of data, verification of statistical methods. All authors read and approved the final manuscript.

\section{Consent for publication}

Not applicable

\section{Ethics approval and consent to participate}

All clinical data in this case report were either provided by the patient or collected by our team's members with the consent of them. There was no additional invasive test or experimental drugs used out of order for the patient. This study was approved by the ethics committee of Fujian Medical University Union Hospital and written consent was obtained from all patients involved.

\section{Author details}

${ }^{1}$ Department of Neurology, Fujian Institute of Geriatrics, Fujian Medical University Union Hospital, Fuzhou, China. ${ }^{2}$ Department of Neurology, Quanzhou First Hospital Affiliated to Fujian Medical University, Quanzhou, China. ${ }^{3}$ Department of Radiology, Fujian Medical University Union Hospital, Fuzhou, China.

${ }^{4}$ Key Laboratory of Brain Aging and Neurodegenerative Diseases, Fujian Key Laboratory of Molecular Neurology, Fujian Medical University, Fuzhou, China.

\section{Publisher's Note}

Springer Nature remains neutral with regard to jurisdictional claims in published maps and institutional affiliations

\section{References}

1.Lozano R, Naghavi M, Foreman K, Lim S, Shibuya K, Aboyans V, Abraham J, Adair T, Aggarwal R, Ahn SY et al: Global and regional mortality from 235 causes of death for 20 age groups in 1990 and 2010: a systematic analysis for the Global Burden of Disease Study 2010. The Lancet 2012, 380(9859):20952128. 
2.B F, G N, J P, B T, G T, E D-T: European Stroke Organisation (ESO) guidelines on glycaemia management in acute stroke. European stroke journal 2018, 3(1):5-21.

3.Smith CJ, Emsley HC, Libetta CM, Hughes DG, Drennan RF, Vail A, Tyrrell PJ: The Oxfordshire Community Stroke Project classification in the early hours of ischemic stroke and relation to infarct site and size on cranial computed tomography. Journal of Stroke \& Cerebrovascular Diseases 2001, 10(5):205-209.

4.Lima AN, Bittar R, Gattas GS, Borsengshu E, Oliveira ML, Monsanto R, Bittar LF: Pathophysiology and Diagnosis of Vertebrobasilar Insufficiency: A Review of the Literature. International Archives of Otorhinolaryngology 2017, 21(3):302-307.

5.† JAD, Leigh JS, Williams DS, Koretsky AP: Perfusion imaging. Magnetic Resonance in Medicine 1992, 23(1):37-45.

6.Lou X, Cai YQ, Lin MA, Cai JM, Ning-Yu AN, Xue-Mei LI: ASL MRI for Cerebral Hypoperfusion in Patients with Symptomatic Carotid Artery Occlusion. Chinese Journal of Medical Imaging 2007.

7.Peng Q, Yaoguang HU, Changqing YE, Bing WU, Huang Y, Neurology DO: A comparison study of TCD vs MRI VE-ASL in the evaluation of collateral circulation of cerebral arteries. Chinese Journal of Nervous \& Mental Diseases 2014.

8.Clement P, Mutsaerts HJ, Vã cL, Ghariq E, Pizzini FB, Smits M, Acou M, Jovicich J, Vanninen R, Kononen M: Variability of physiological brain perfusion in healthy subjects - A systematic review of modifiers. Considerations for multi-center ASL studies. J Cereb Blood Flow Metab 2017:271678X17702156.

9. Hua Y, Tao YL, Li M, Yong Q, He W, Zhao H, Luo Y, Zhang Y, Peng T, Yu DL: Multicenter ultrasound screening for the results of carotid atherosclerotic lesions in a Chinese population with high-risk of stroke: A preliminary analysis. Chinese Journal of Cerebrovascular Diseases 2014, 11(12):617-623.

10.LR C, RJ W, TA G, J T, L P, HM C, P T, JF D, CJ C, JC B et al: New England medical center posterior circulation registry; 2004.

11.Cosar M, Yaman M, Eser O, Songur A, Ozen OA: Basilar artery angulation and vertigo due to the hemodynamic effect of dominant vertebral artery. Medical Hypotheses 2008, 70(70):941-943.

12.M A, C G, KK A, TE W, KSG C, CWK K, GJ EJ, KS P, C W: Facilitating motor imagery-based braincomputer interface for stroke patients using passive movement. Neural computing \& applications 2017, 28(11):3259-3272.

13.J L, D W: Advances in endovascular therapy for ischemic cerebrovascular diseases. Chronic diseases and translational medicine 2016, 2(3):135-139. 
14.Listed N: Summaries for patients. Screening for suicide risk in adolescents, adults, and older adults in primary care: recommendations from the U.S. Preventive Services Task Force. Annals of Internal Medicine 2014, 160(10):I.

15.Prati P, Tosetto A, Vanuzzo D, Bader G, Casaroli M, Canciani L, Castellani S, Touboul PJ: Carotid intima media thickness and plaques can predict the occurrence of ischemic cerebrovascular events. Stroke; a journal of cerebral circulation 2008, 39(9):2470.

16.Polak JF, Pencina MJ, O'Leary DH, D'Agostino RB: Common carotid artery intima-media thickness progression as a predictor of stroke in multi-ethnic study of atherosclerosis. Stroke; a journal of cerebral circulation 2011, 42(11):3017.

17.Jiang Y, Guo H, Zhou Y: The Comparison of rCBF and MRI in the Senile Patients with Acute Ischemic Cerebrovascular Disease. Chinese Journal of Medical Imaging Technology 2000.

\section{Tables}

Table 1: General clinical data of each group of personnel

\begin{tabular}{|c|c|c|c|c|}
\hline \multirow[t]{2}{*}{ Variable } & \multicolumn{2}{|c|}{ Stroke high risk group } & \multirow{2}{*}{$\begin{array}{c}\text { Total number of high risk } \\
\text { groups }\end{array}$} & \multirow[t]{2}{*}{$\mathrm{p}$} \\
\hline & $\begin{array}{c}\text { Vertebral artery stenosis } \\
\text { group }\end{array}$ & $\begin{array}{l}\text { Without Vertebral artery } \\
\text { stenosis group }\end{array}$ & & \\
\hline Total & 20 & 23 & 43 & \\
\hline Gender & & & & 0.126 \\
\hline male & 10 & 16 & 26 & \\
\hline female & 10 & 7 & 17 & \\
\hline Age(years) & $62.70 \pm 11.77$ & $67.04 \pm 9.01$ & $64.81 \pm 10.52$ & 0.645 \\
\hline LVAT & 7 & 0 & 7 & - \\
\hline RVAT & 11 & 0 & 11 & - \\
\hline BVAT & 2 & 0 & 2 & - \\
\hline
\end{tabular}

LVAT: Left vertebral artery stenosis

RVAT: Right vertebral artery stenosis

BVAT: Bilateral vertebral artery stenosis stenosis

Table 2םBI and CBF value of brain regions in the VAS group and the non-VAS group 


\begin{tabular}{|c|c|c|c|}
\hline Variable & $\begin{array}{c}\text { the VAS group } \\
\square \mathrm{n}=20 \square(\mathrm{ml} / 100 \mathrm{~g} / \mathrm{min})\end{array}$ & the non-VAS group $\square \mathrm{n}=23 \square(\mathrm{ml} / 100 \mathrm{~g} / \mathrm{min})$ & $P$ \\
\hline Frontal lobe BI value & $7.38 \pm 5.43$ & $6.46 \pm 3.76$ & 0.518 \\
\hline Parietal BI value & $6.71 \pm 5.52$ & $5.02 \pm 4.35$ & 0.268 \\
\hline Occipital BI value & $14.00 \pm 4.54$ & $5.65 \pm 5.00$ & 0.000 \\
\hline Temporal lobe BI value & $5.44 \pm 4.17$ & $5.89 \pm 5.03$ & 0.756 \\
\hline Cerebellar BI value & $4.64 \pm 4.64$ & $4.82 \pm 3.56$ & 0.888 \\
\hline Basal ganglia BI value & $3.52 \pm 3.42$ & $4.82 \pm 4.08$ & 0.279 \\
\hline Thalamic BI value & $6.37 \pm 4.74$ & $6.50 \pm 5.46$ & 0.930 \\
\hline Hippocampal BI value & $7.13 \pm 5.96$ & $7.51 \pm 5.74$ & 0.836 \\
\hline Left Frontal lobe CBF value & 41.2716 .65 & 40.0013 .12 & 0.781 \\
\hline Left Parietal CBF value & 32.1119 .34 & 29.3211 .81 & 0.580 \\
\hline Left Occipital CBF value & 38.6414 .52 & 35.928 .57 & 0.468 \\
\hline Left Temporal lobe CBF value & 36.2641 .03 & 31.657 .78 & 0.183 \\
\hline Left Cerebellar CBF value & 38.9719 .23 & 41.2016 .18 & 0.683 \\
\hline Left Basal ganglia CBF value & 49.0411 .80 & 52.4511 .01 & 0.340 \\
\hline Left Thalamic CBF value & 54.2525 .36 & 49.3417 .15 & 0.456 \\
\hline Left Hippocampal CBF value & 51.4521 .24 & 50.107 .97 & 0.789 \\
\hline Right Frontal lobe CBF value & 36.9818 .87 & 36.7013 .68 & 0.875 \\
\hline Right Parietal CBF value & 34.5020 .40 & 31.2511 .75 & 0.955 \\
\hline Right Occipital CBF value & 42.4011 .41 & 38.007 .05 & 0.535 \\
\hline Right Temporal lobe CBF value & 35.3211 .33 & 32.629 .16 & 0.130 \\
\hline Right Cerebellar CBF value & 39.7319 .46 & 39.0714 .96 & 0.394 \\
\hline Right Basal ganglia CBF value & 51.0113 .06 & 52.529 .40 & 0.901 \\
\hline Right Thalamic CBF value & 53.1524 .54 & 50.9914 .40 & 0.662 \\
\hline Right Hippocampal CBF value & 55.6123 .61 & 50.9812 .14 & 0.722 \\
\hline Mean value of $\mathrm{LCH}$ & 334.80120 .95 & 329.9775 .37 & 0.414 \\
\hline Mean value of $\mathrm{RCH}$ & 348.70114 .91 & 332.1272 .98 & 0.570 \\
\hline
\end{tabular}

LCH: Left cerebral hemisphere

RCH: Right cerebral hemisphere

Table 3: Cut-off values of BI values on the left side of the occipital lobe Test result variable Area under curveStandard error $\quad P \quad 95 \% \pm C I$ Cut-off values

\begin{tabular}{llllc}
\hline Occipital lobe BI & 0.887 & 0.062 & $\square 0.010 .789-0.985$ & 10.46 \\
Occipital lobe ВIロ10 & 0.860 & 0.050 & $\square 0.010 .738-0.982$ & - \\
\hline
\end{tabular}

Table 4: Verification of cut-off values for BI values on the left side of the occipital region 


\begin{tabular}{|c|c|c|c|c|c|c|}
\hline Variable & the VAS group $\square n=20 \square$ & $\begin{array}{c}\text { the non-VAS group } \\
\square n=23 \square\end{array}$ & $P$ & OR & $95 \% \pm \mathrm{CI}$ & $P$ \\
\hline OLBI $\geq 10.46$ & & & 0.000 & 15.776 & $1.204-206.683$ & 0.036 \\
\hline Yes & 18 & 6 & & & & \\
\hline No & 2 & 17 & & & & \\
\hline
\end{tabular}

Occipital lobe CI; Occipital lobe CI

Figures

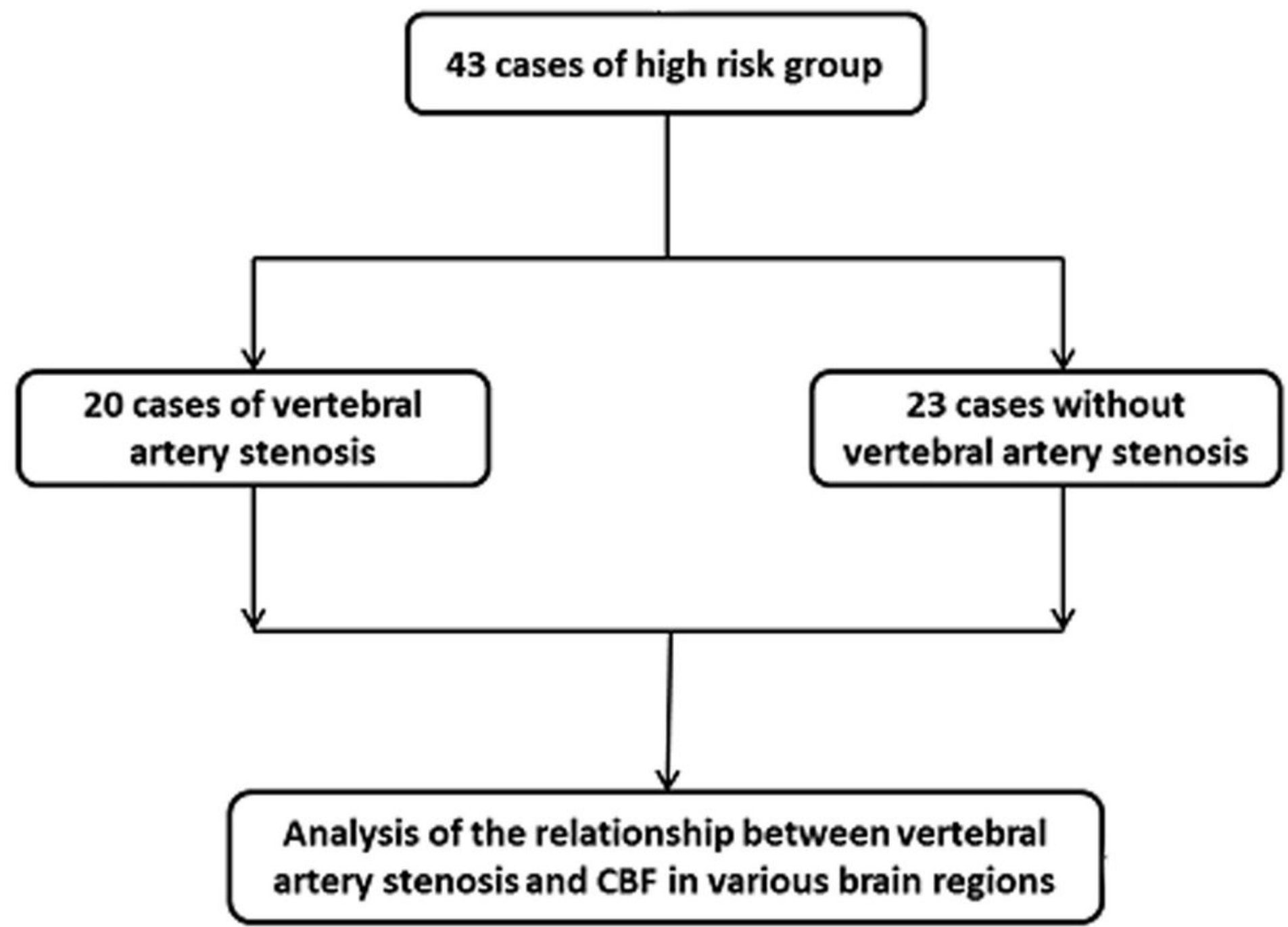

Figure 1

Diagram of study population 


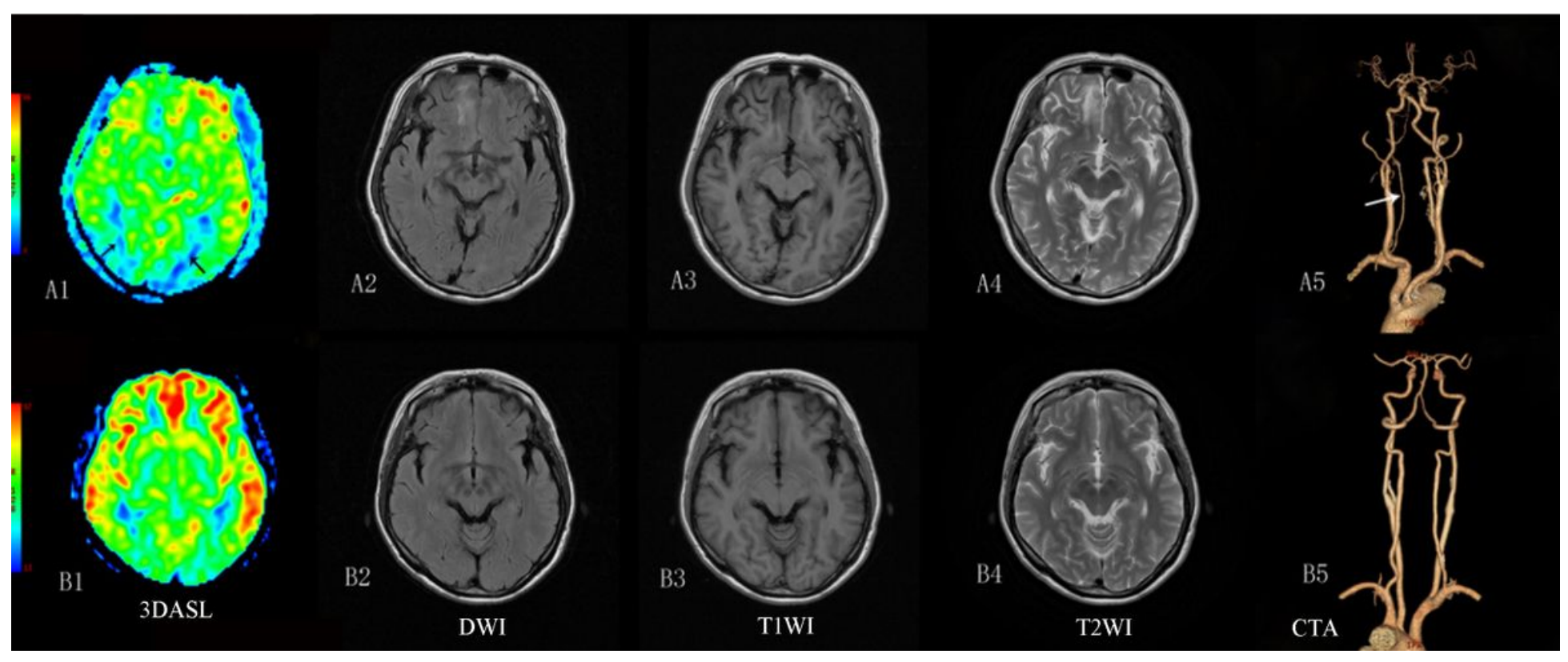

\section{Figure 2}

Images of the VAS group and the non-VAS group. A1: The 3D-ASL image of the VAS group. The posterior circulatory brain area (especially the occipital lobe) was significantly decreased (black arrowhead). A2】 There was no obvious acute lesion in the DWI image of the VAS group.A3囚T1-weighted image of the VAS group. A4囚T2-weighted images of the VAS group. A5『CTA images of the VAS group with fine vertebral artery stenosis (white arrow). B1】ln the non-VAS group, no significant decrease of perfusion was observed on 3d-ASL images. B2囚In the non-VAS group, there was no obvious acute lesion on DWI images. B3囚T 1 weighted images of the non-VAS group.B4 $\triangle \mathrm{T} 2$ weighted images of the non-VAS group. B5『In the non-VAS group, no obvious stenosis was found on CTA images. 


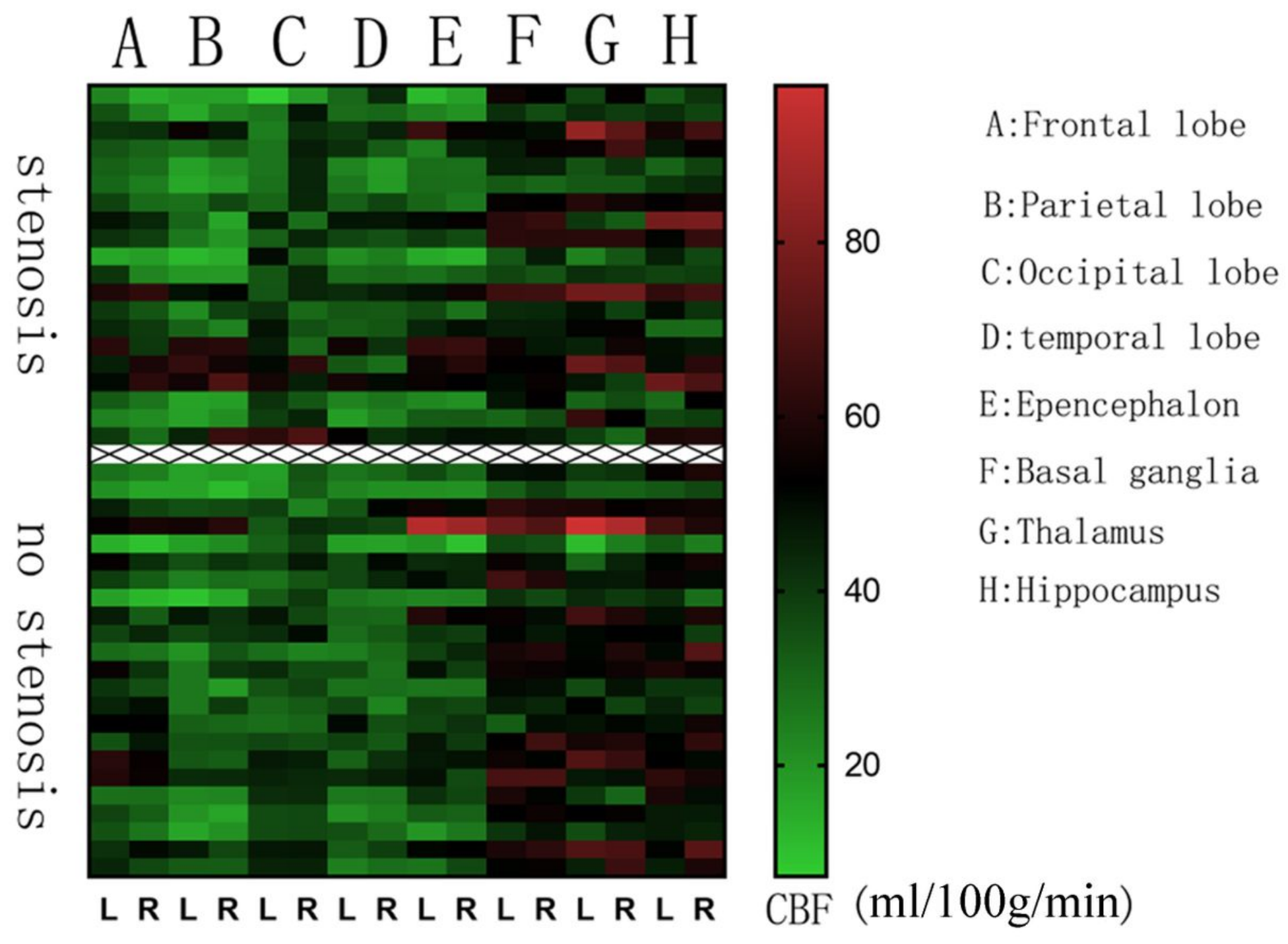

Figure 3

CBF values for the brain regions of interest (frontal, parietal, temporal, occipital, cerebellum, hippocampus, basal ganglia, and thalamus) in patients at risk of stroke and vertebral artery stenosis. The CBF values of the thalamus, basal ganglia and hippocampus were higher than those of other brain regions. 


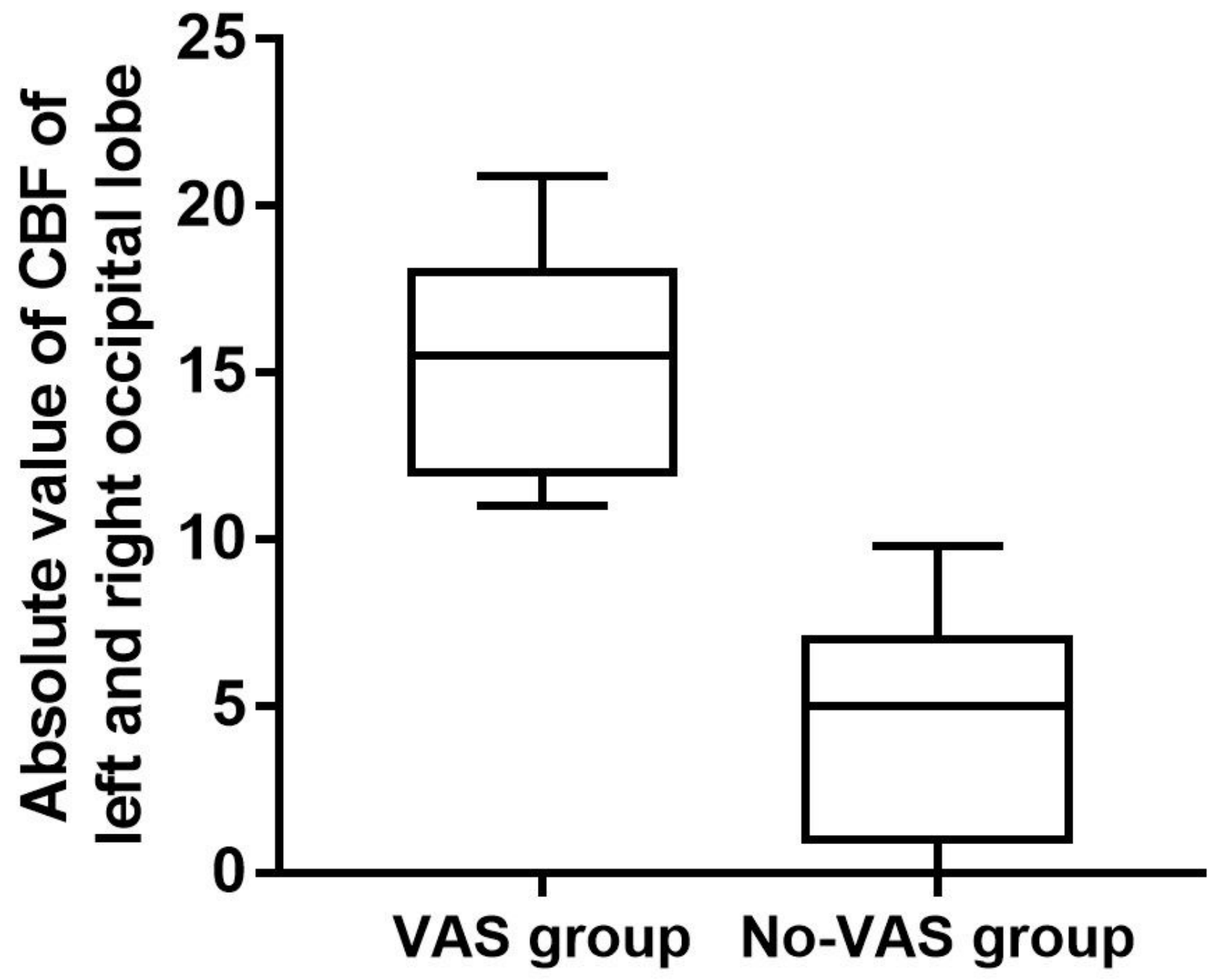

Figure 4

The BI values of the VAS group and the non-VAS group represented as box plots. Horizontal lines represent the median, the bottom and top of the boxes represent the 25th and 75th percentiles, respectively, and the vertical bars represent the range of data. 


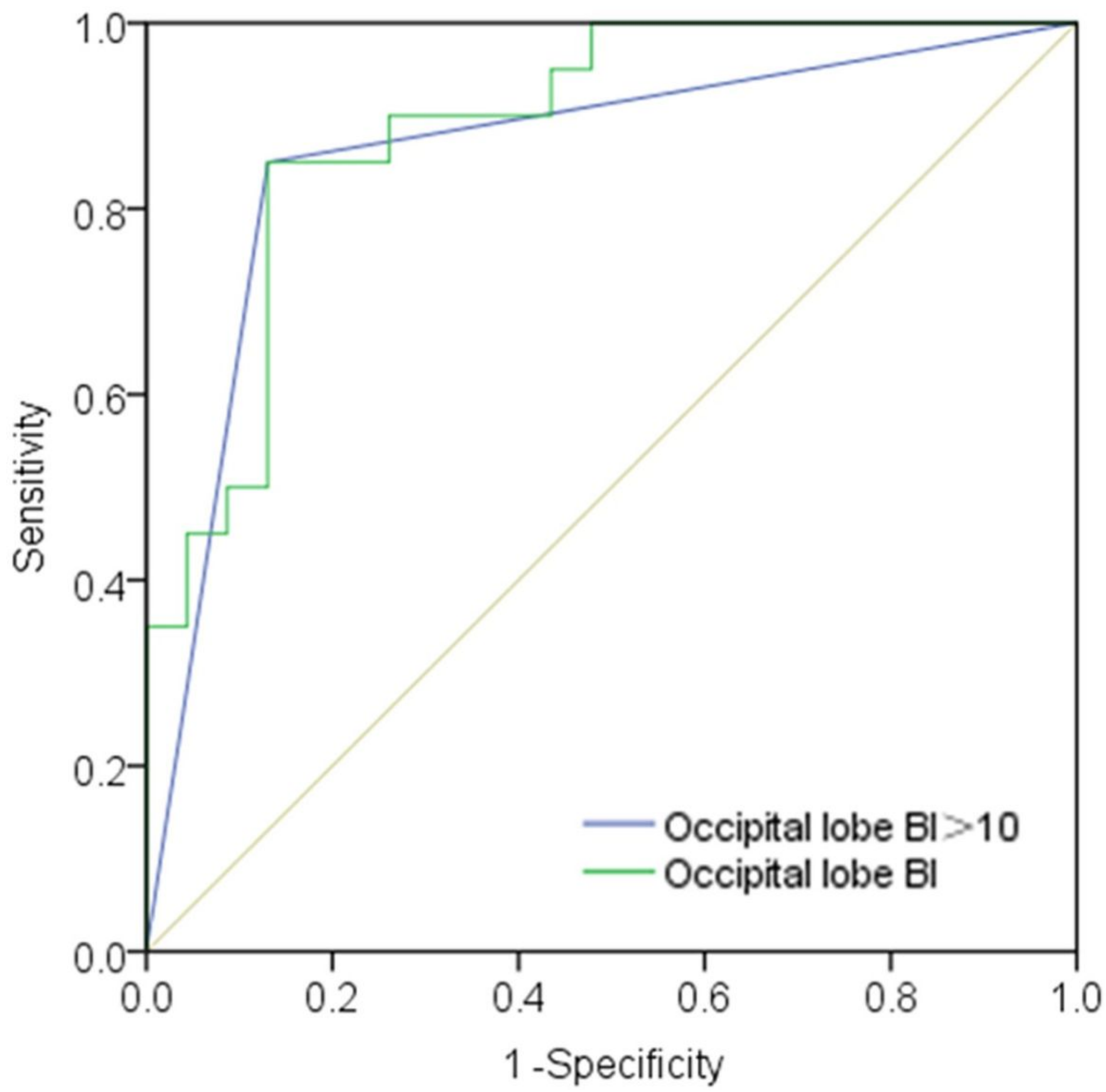

Figure 5

ROC curve of BI values on the occipital lobe. According to the binary logistic regression results, the ROC curves of the BI values of the occipital lobe are shown.

\section{Supplementary Files}

This is a list of supplementary files associated with this preprint. Click to download.

- Supplementaryfigure1.jpg 
- Supplementaryfigure2.tif

Page 18/18 